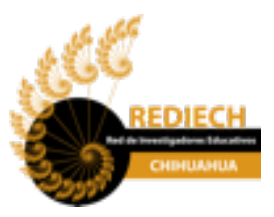

Red de Investigadores Educativos Chihuahua A.C. Chihuahua, México www.rediech.org

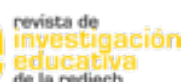

de la rediech

ISSN: 2007-4336

ISSN-e: 2448-8550

http://www.rediech.org/ojs/2017/index.php/ie rie rediech/index

\title{
EDUCACIÓN PARA LA EMPLEABILIDAD: ENFOQUE DE LA INVESTIGACIÓN EDUCATIVA
}

IE Revista de Investigación Educativa de la REDIECH, 10(19), pp. 221-238.

DOI: http://dx.doi.org/10.33010/ie_rie_rediech.v10i19.715

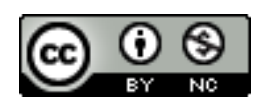

Esta obra está bajo licencia internacional

Creative Commons Reconocimiento-NoComercial 4.0.

CC BY-NC 4.0 


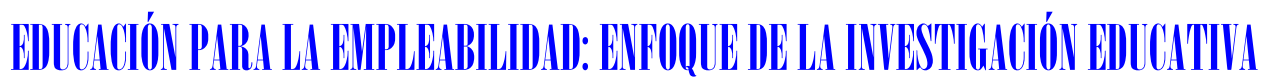

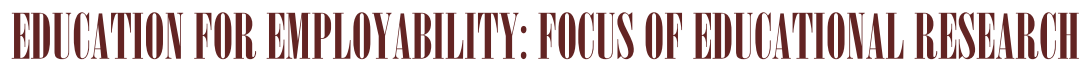

\author{
DÍAZ GÓMEZ Eduardo Raúl \\ Recepción: junio 20 de 2019 | Aprobado para publicación: septiembre 29 de 2019 \\ DOI: http://dx.doi.org/10.33010/ie_rie_rediech.v10il9.715
}

\section{Resumen}

Existe interés en círculos académicos por discutir el papel de las universidades en la preparación de estudiantes para el empleo y desarrollo de competencias para la empleabilidad. Este interés parte de los altos índices de desempleo entre jóvenes profesionistas en algunos países. Este artículo tiene como propósito documentar avances logrados respecto a la investigación educativa con enfoque en empleabilidad. Con base en una metodología cualitativa de análisis de contenido, se examinaron 46 artículos arbitrados sobre educación para la empleabilidad. Los resultados señalan seis categorías de temas en los trabajos de la muestra respecto a la labor que se lleva a cabo en universidades para fomentar la empleabilidad. Estas categorías son: competencias transversales para la empleabilidad, equidad, fomento a la empleabilidad, internacionalización, programas educativos y mercado laboral. Estas seis categorías representan oportunidades para la docencia, servicio institucional e investigación. Las implicaciones se discuten desde la perspectiva de las aportaciones de Boyer y otros que argumentan en favor de la articulación de funciones del profesorado.

Palabras clave: EDUCACIÓN, EMPLEABILIDAD, COMPETENCIAS, INVESTIGACIÓN, DOCENCIA.

\footnotetext{
Abstract

There is interest within academic circles in discussing the role of universities in preparing students for employment and the development

Eduardo Raúl Díaz Gómez. Profesor de tiempo completo en la Escuela de Administración y Negocios Cetys Universidad, Tijuana, BC, México. Doctor en liderazgo con concentración en liderazgo organizacional. Autor de varios artículos, incluyendo "Leadership self-efficacy: A study of male and female MBA students in Mexico" en la revista Advancing Women in Leadership. Evaluador para la Federación de Instituciones Mexicanas Particulares de Educación Superior (FIMPES). Correo electrónico: eduardo.diaz@cetys.mx. ID: https://orcid.org/0000-0003-0053-8751.
} 
of employability competencies. This interest developed from the high unemployment rates of recent college graduates in some countries. The purpose of the article was to support this discussion and frame it in terms of the achievements of scholars involved in this type of research. Under a qualitative content analysis approach, 46 articles on employability were examined. The analysis emerged six major themes about the work that universities engage in to promote employability. These categories are: cross-discipline competencies, equity, employability promotion, internationalization, educational programs, and labor market. These categories represent teaching, service, and research opportunities for teachers and academics. Implications are discussed in terms of the contributions attributed to Boyer and others who argued in favor of articulating the functions of the professoriate.

Keywords: education, employability, competencies, research, teaching.

\section{IITrinonccóín}

El personal docente en las instituciones de educación superior (IES) tiene la tarea de transmitir conocimientos a sus alumnos, ayudarles a desarrollar las competencias que necesitan para la vida y el trabajo y crear nuevo conocimiento a través de publicaciones científicas. Desde hace 30 años, las ideas de Ernest Boyer han servido como guía para los docentes que integran su labor en el aula con actividades de servicio institucional e investigación educativa. Boyer et al. (2016) argumentaron que la investigación educativa debe contribuir al proceso de enseñanza-aprendizaje de los estudiantes. Esto implica que los profesores universitarios basen, en parte, su labor de investigación en las experiencias y actividades de clase promoviendo la implementación de estrategias educativas innovadoras mientras contribuyen al desarrollo de la investigación en educación. Este enfoque ha sido ampliamente denominado scholarship of teaching and learning (SoTL), utilizando la terminología en inglés. Los trabajos de Bonney (2018) y de Bernstein (2018) son buenos ejemplos de la adopción de este enfoque de la investigación educativa.

El actual estudio tiene como propósito revisar los avances en la investigación respecto al papel de las IES en promover la empleabilidad de sus egresados. A través del análisis de contenido de artículos publicados en revistas académicas, se identificaron resultados de investigaciones que documentan la aplicación de estrategias de enseñanza-aprendizaje orientadas a promover la empleabilidad de individuos durante sus estudios universitarios. En congruencia con planteamientos basados en SoTL, los artículos revisados dan muestra de trabajos de investigación que parten de actividades curriculares y cocurriculares donde los investigadores participan junto con sus alumnos durante el proceso de investigación, o bien documentan las experiencias de los estudiantes, personal administrativo y académico de las IES. Los presentes hallazgos serán de utilidad para personal administrativo y docente en IES interesados 222 en promover la empleabilidad de sus estudiantes y la investigación educativa, así 
como agilizar procesos de acreditación institucional o a nivel de programa académico. Para fines de este estudio, el término empleabilidad se utiliza para referirse a las competencias relacionadas con la capacidad de los estudiantes y egresados de las IES para colocarse en un empleo y perseverar en el trabajo a lo largo del tiempo. Asimismo, las actividades de docencia se refieren al trabajo del personal académico impartiendo clases, mientras que el servicio institucional corresponde a trabajos de soporte en procesos de acreditación, coordinaciones de programas, tutorías y diferentes servicios en apoyo directo o indirecto con estudiantes.

\section{Justificiación del estudio}

Milner, Cousins y McGowan (2016) exhortaron a la comunidad académica a explorar temas relacionados con el rol de las IES en promover la empleabilidad de sus egresados. Asimismo, Fernández Fassnacht (2017) argumentó que las IES en México enfrentan el reto de demostrar que siguen siendo relevantes y recomendó a los líderes educativos asegurar que los planes de estudio y proyectos de investigación educativa se lleven a cabo en función de las necesidades del entorno. Adicionalmente, Wolff y Booth (2017) argumentaron que las IES se encuentran ante la necesidad de decidir cómo fomentar y evaluar el desarrollo de competencias para la empleabilidad en sus estudiantes. Los autores comentaron que, sin duda, las personas responsables por el desarrollo de los estudiantes universitarios buscarán ayudarles para que se conviertan en profesionistas que se coloquen con facilidad en empleos bien remunerados; sin embargo, consideraron arriesgado suponer que los líderes educativos y docentes saben qué competencias se alinean con la empleabilidad de sus alumnos.

El problema del desempleo en jóvenes recién egresados de IES está documentado y tiene lugar en diferentes países como Estados Unidos (Cunningham, 2016), Chile (Arias, 2018), México (Moctezuma et al., 2013) y algunos países europeos (Eliška, 2016; Chadha y Toner, 2017). En todos estos casos, los jóvenes que pertenecen a grupos de bajo nivel socioeconómico tienden a ser los más afectados. En el caso particular de México, el problema se debe en parte a la desarticulación entre las políticas públicas en materia de educación y las industrias con alto potencial para la competitividad (Licona Michel y Rangel Delgado, 2013), así como a la reducción de la calidad del empleo en ciertas regiones del país (Hualde y Serrano, 2005) y el bajo desempeño en el desarrollo de competencias para el trabajo por parte de los egresados de IES (Sueyoshi y Rangel Delgado, 2016).

Fomentar la empleabilidad en estudiantes y egresados de IES requiere de esfuerzo por parte de diferentes actores. Ciertamente requiere de la pericia y disposición del personal docente, pero también es necesario contar con apoyo administrativo, involucramiento por parte de representantes de la industria y compromiso de los estudiantes. En el centro de todo se encuentra la investigación educativa, a través de la cual los investigadores promueven la discusión que guía a los diferentes actores relacionados con IES para que generen políticas y acciones congruentes con un propósito común (Pfleger et al., 2018).

Al momento se cuenta con pocos estudios diseñados para examinar este tipo de investigación educativa. Entre los que es conveniente señalar se encuentra el trabajo 
de Chadha y Toner (2017), donde se llevó a cabo un análisis de contenido de los sitios de internet de diferentes IES en Estados Unidos y Reino Unido con el propósito de identificar cómo estas instituciones promueven sus compromisos con la empleabilidad de sus alumnos. Los hallazgos del estudio sugieren que, en Reino Unido, las IES se alinean con el discurso del gobierno en sus promesas de preparar estudiantes para el empleo, mientras en Estados Unidos las IES se basan más en la redacción de sus propias misiones y visiones que se enfocan en el desarrollo del capital humano.

Existe evidencia respecto a la urgencia de este tipo de investigación. El informe del Plan Nacional de Evaluación de los Aprendizajes (Planea) advierte que los egresados de educación media superior están muy limitados en términos de sus habilidades de lenguaje, comunicación y matemáticas (Backhoff Escudero et al., 2018). Este rezago afecta el desenvolvimiento del estudiante en la universidad, ya que las habilidades para comunicarse de manera efectiva y la capacidad de razonamiento cuantitativo son esenciales para el desarrollo de competencias transversales características de profesionales exitosos. La demanda de profesionistas en el mercado laboral en México es uno de los principales criterios en que se basan los estudiantes al momento de elegir una carrera profesional (Moctezuma et al., 2013). Sin embargo, la inversión en educación superior muestra rendimientos por debajo de las expectativas de los estudiantes y sus familias (De la Luz Tovar y Díaz González, 2010).

En opinión de algunos autores, las IES no deben estar exentas de rendir cuentas sobre su impacto en la industria mediante la contribución de profesionistas que reúnan las características de la demanda en el mercado laboral (Wolff y Booth, 2017; Chadha y Toner, 2017). En este sentido, no es de sorprender que, según la Encuesta Nacional de Ocupación y Empleo, en el primer trimestre del 2018 más de 807 mil personas con niveles educativos de educación media superior y superior en México se encontraban desempleados (INEGI, 2018). Este tipo de situaciones lleva a cuestionar el trabajo que se realiza en las IES y la calidad de educación que reciben los estudiantes (Moctezuma et al., 2013).

En México se han tomado medidas para intentar mantener un estándar de calidad educativa. Jiménez Moreno (2017) describió el proceso comenzado a partir de la década de 1980 cuando las IES adoptaron el examen general para el egreso de la licenciatura (EGEL) como un mecanismo para la evaluación de programas educativos y facilitador para la rendición de cuentas a la sociedad sobre el desempeño de las instituciones educativas.

El EGEL resultó ser un instrumento de control adecuado (López Aguado, 2018), pero dada la masificación de la educación que ha tenido lugar desde hace más de 30 años, el gobierno federal comenzó la implementación de reformas diseñadas para mejorar la calidad de servicios educativos, enfocándose principalmente en la preparación de los egresados y su capacidad para conseguir empleo (Moctezuma et al., 2013). Sin embargo, Martínez Lobatos (2014) analizó la opinión de estudiantes de profesional en Baja California para conocer su percepción respecto a la efectividad de sus programas educativos en términos de preparación para el trabajo mediante experiencias fuera del aula. La autora concluyó que era necesario dar prioridad a este objetivo y dejar de posponer la implementación de prácticas educativas enfocadas

224 al desarrollo de competencias en contextos profesionales. 
En este artículo se documentan investigaciones sobre prácticas docentes y programas educativos que se alínean con la meta de preparar estudiantes universitarios para el empleo. En congruencia con el enfoque SoTL, se parte del supuesto de que la educación dirigida al fomento de habilidades para la empleabilidad se caracteriza por el trabajo de personal docente consciente de la demanda del mercado y capaz de mantener rigor académico en el aula (Udall, Forrest y Stewart, 2015). En concreto, este estudio tiene dos aportaciones. Primero, apoya a la industria de la educación ante la creciente presión por cumplir con las expectativas de la comunidad respecto a la empleabilidad de los egresados de IES (Chadha y Toner, 2017). Segundo, revisa las contribuciones a la investigación sobre empleabilidad bajo un enfoque congruente con SoTL originalmente propuesto por Boyer para señalar la congruencia entre la docencia, el servicio institucional y la investigación educativa.

\section{Là reconsiderarición eduruativia de Boyẹer}

Boyer provocó controversia y discusión cuando criticó a la comunidad académica acostumbrada a reconocer el desempeño de los docentes con base en su labor como investigadores, ignorando sus actividades de servicio y enseñanza (Boyer et al., 2016). La principal preocupación de Boyer radicaba en los incentivos para docentes que los enfocaban más en la investigación, pero que los alejaban de la formación de sus estudiantes. Al momento de la primera edición de su libro, Boyer percibía que los profesores en IES que aspiraban a una posición de tiempo completo y mejor remuneración y prestigio tenían que ganárselo mediante su labor de investigación, sin importar realmente su desempeño dentro del aula. La investigación educativa tiende a ser muy absorbente, por lo que los docentes se ven en la necesidad de elegir entre avanzar en sus carreras o dedicar el tiempo suficiente a sus estudiantes para que egresen de la universidad mejor preparados (Boyer et al., 2016).

Desde la primera publicación de los argumentos centrales de Boyer en 1990, otros académicos han continuado la discusión. Caballero y Bolívar (2015) argumentaron que la educación universitaria debe ser modificada de manera que se integre la docencia, el aprendizaje y la investigación. En este sentido, los docentes tienen oportunidad de aplicar métodos distintos para fomentar en sus estudiantes aprendizajes significativos que puedan llevar a la práctica y de esa forma mejorar su desempeño profesional. Posteriormente, los docentes podrían evaluar, discutir y divulgar los resultados de su trabajo mediante revistas académicas con la finalidad de permitir a otros conocer su hallazgos y experiencias y replicar mejores prácticas. Al mismo tiempo, los docentes obtienen crédito por sus publicaciones, mejorando su estatus como investigadores y su posición dentro de sus instituciones educativas. Como beneficio adicional, los estudiantes se convierten en participantes en algunos proyectos de investigación de sus maestros, desarrollando competencias genéricas importantes, como el razonamiento lógico y la argumentación, que tienen aplicación para el trabajo.

Por su parte, Kreber (2002) explicó que la diferencia entre docentes y docentes-investigadores radica en que los segundos tienen la capacidad de enseñar, pero también saben documentar su trabajo en el aula de manera que sea publicable en 
revistas arbitradas. Estos docentes-investigadores necesitan el apoyo de líderes educativos para desarrollar su trabajo de manera más efectiva. En este sentido, Mathany, Clow y Aspenlieder (2017) argumentaron que apoyar la investigación y docencia implica apoyar a aquellos interesados en fomentar el aprendizaje. Claramente, los seguidores de Boyer perciben la articulación de la docencia y la investigación como parte de una cultura de calidad institucional planeada. La creación de una cultura de calidad en la educación involucra una justificación del trabajo académico, el proceso planeado del desarrollo de los estudiantes y la adaptación a las demandas cambiantes de la sociedad (Yingqiang y Su, 2016). Estas ideas han sido aceptadas con el paso del tiempo y actualmente sirven como base para una línea de investigación importante. Divan et al. (2017) analizaron 223 artículos publicados entre 2012 y 2014 en las tres principales revistas sobre SoTL (International Journal for the Scholarship of Teaching and Learning, International Journal of Teaching and Learning in Higher Education y el Journal of the Scholarship of Teaching and Learning). Los autores concluyeron que existe un balance en los enfoques metodológicos (cualitativo, cuantitativo, métodos mixtos) de los artículos y que la mayor parte de los participantes en las investigaciones son estudiantes. La existencia de estas publicaciones de prestigio constituye evidencia del interés que ha generado la crítica de Boyer en países desarrollados, pero todavía no se alcanza a percibir el mismo grado de interés en países emergentes.

El enfoque educativo de Boyer sugiere que las divisiones entre la docencia, investigación y administración educativa deben ser porosas, para permitir la creación de un sistema organizacional que facilite el cumplimiento de metas de mayor orden que los objetivos específicos por funciones o departamentos organizacionales. Este tipo de organización es congruente con los planteamientos que caracterizan a las llamadas organizaciones que aprenden (Senge et al., 2012). Fomentar culturas educativas que evolucionen a través de sus experiencias implica implementar un estilo de liderazgo relajado sobre los cuerpos colegiados o academias, lo cual sucede por tradición en los entornos educativos (Huisman y Currie 2004). Principalmente, preparar estudiantes para la empleabilidad y para la vida en general requiere de un enfoque sistémico donde intervienen diferentes actores de la comunidad, la escuela, la industria y el hogar (DiBenedetto y Brian Myers, 2016).

Siguiendo los planteamientos originalmente propuestos por Senge et al. (2012), varios autores han desarrollado investigaciones basadas en la documentación de experiencias educativas de manera congruente con el enfoque en sistemas (Ison, 1999; Davis, Dent y Wharff, 2015; Dunnion y O’Donovan, 2014). En el entorno de la educación superior existen diferentes retos importantes que llevan a las IES a evolucionar de forma planeada (Kezar, 2005), situación que justifica la adopción de un esquema de administración basado en sistemas en IES.

Banathy (1999) argumentó que el aprendizaje requiere de aplicación, lo que sugiere un enfoque en sistemas donde la docencia incluye objetivos de aprendizaje, métodos de enseñanza, contextos relevantes para la aplicación del conocimiento, integración del conocimiento en contextos más amplios y la creación de nuevo conocimiento en función de lo aprendido. Bajo el modelo de Senge et al. (2012), el 226 análisis y la reflexión sobre estas actividades lleva al planteamiento de estrategias 
de mejora continua. El cambio planeado en las IES ha comenzado en diferentes partes del mundo. Las universidades en Europa comienzan a mostrase más emprendedoras, caracterizadas por culturas organizaciones que favorecen la diversidad de unidades académicas, fuentes de ingresos y barreras organizacionales porosas (Van Der Meulen, 2002). Incluso hay evidencia de acciones planeadas para crear una cultura de aprendizaje organizacional en las universidades. Ponnuswamy y Lysander (2016) encuestaron a 700 docentes en la India y encontraron que existen relaciones significativas entre la cultura organizacional enfocada al aprendizaje y desempeño enfocado al conocimiento y la investigación.

Lo anterior sugiere que es posible articular la docencia, servicio institucional e investigación educativa, pero requiere de una cultura organizacional en IES conducente a la mejora continua, rendición de cuentas y libertad para colaborar a través de barreras organizacionales. Esto está sucediendo en diferentes lugares, pero las ideas de Boyer no han permeado lo suficiente en países emergentes. Sin embargo, es importante articular las funciones del profesorado y enfocarlas al cumplimiento de ciertos objetivos, incluyendo el fomento a la empleabilidad, para que las IES mantengan su relevancia. Con esto se busca dar respuesta a las interrogantes que se plantean a continuación.

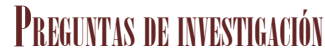

1. ¿Cuáles son los temas principales encontrados en la literatura académica respecto a la empleabilidad de egresados de las IES?

2. ¿Qué prácticas o experiencias educativas tienen impacto en la promoción de la empleabilidad de egresados universitarios?

3. ¿Cómo se aplican las premisas de SoTL en la literatura sobre empleabilidad?

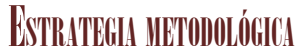

Este artículo fue desarrollado con base en una metodología de análisis cualitativo de análisis de contenido congruente con el procedimiento descrito por Gervilla Castillo (2004), así como White y Marsh (2006) y el análisis de contenido llevado a cabo por Bowen (2010) en un estudio similar. Tomando como ejemplo la descripción del método de análisis de contenidos propuesto por Gervilla Castillo (2004), las fases de la investigación del presente estudio se llevaron a cabo en el siguiente orden:

1. Elección del contenido o definición del universo. El acopio de artículos se llevó a cabo a través de la base de datos EBSCO Host durante los meses de junio y julio de 2018. Los filtros establecidos fueron incluir únicamente textos completos y de artículos arbitrados. No se utilizaron filtros de temporalidad, publicaciones 0 geografía para permitir un mayor número de artículos en los resultados. El filtro principal fue utilizar palabras de búsqueda escritas en español, direccionando los resultados hacia trabajos realizados en países de habla hispana, tomando en cuenta que el tema ha sido desarrollado con mayor intención en países como 
Estados Unidos, donde el inglés es la lengua oficial. De esta forma se ayuda parcialmente a controlar aspectos derivados del enfoque cultural (Haasler, 2013).

2. Determinación de los objetivos que se pretenden alcanzar. El objetivo del análisis de contenido fue identificar los temas centrales detrás de las actividades educativas y proyectos de investigación publicados en revistas académicas. Los hallazgos del estudio pueden ser útiles para líderes educativos y personal docente que son responsables por revisar programas académicos, desarrollo curricular 0 la implementación de estrategias educativas en el aula. Asimismo, los resultados sirven a investigadores interesados en explorar el tema de la empleabilidad mediante las líneas de investigación que emergen como resultado de este análisis.

3. Preanálisis. Las combinaciones de búsqueda fueron "empleabilidad" y "educación superior" y también "empleabilidad" y "universidad". La muestra de artículos que finalmente formó parte de este estudio se seleccionó en función de su relevancia con el tema central de estudio a través del análisis de títulos, resúmenes y palabras clave. En algunos casos fue necesario realizar una revisión más minuciosa, ya que los títulos y/o resúmenes estaban redactados de forma ambigua.

4. Codificación. El análisis detallado de los artículos arbitrados permitió identificar temas recurrentes y también temas que superaban el alcance del estudio. Los temas recurrentes se fueron agrupando a lo largo del proceso, mientras que los artículos que daban pie a temas ajenos a la presente investigación fueron descartados. Como resultado, la muestra inicial de 92 artículos pasó a 46 (ver anexo), mismos que fueron segmentados en seis categorías. Las categorías se determinaron con base en la estrategia de codificación in vivo, mediante la cual se utilizan palabras recurrentes en los textos (Saldaña, 2016). Finalmente, cada artículo fue revisado nuevamente con mayor detenimiento en función de sus conclusiones y aportes a la literatura. Con base en los términos y oraciones encontradas con mayor frecuencia en cada categoría se asignaron etiquetas para distinguir entre una categoría y otra. Estas etiquetas son congruentes con categorizaciones encontradas en la literatura sobre educación superior (Saldaña, 2016). El proceso de codificación fue facilitado mediante el uso del software QDA Miner.

\section{Rissurnows}

En total se analizaron 46 artículos arbitrados para identificar los temas principales que abordan y los hallazgos más relevantes en la literatura sobre empleabilidad. Los artículos analizados son congruentes con el enfoque de SoTL, ya que se trata de estudios realizados con base en trabajo docente y/o de servicio institucional que tienen como finalidad promover la empleabilidad entre estudiantes. La referencia completa de cada artículo se encuentra al final de este documento (ver anexo). Se utilizó la estrategia de análisis de datos in vivo (Saldaña, 2016) para etiquetar los temas mejor representados en los artículos (ver tabla 1). Dicha estrategia de análisis de datos en estudios cualitativos consiste en etiquetar los temas emergentes de los

228 textos con frases o palabras utilizadas en los textos analizados. 


\begin{tabular}{lc}
\hline Tabla 1. Categorías de temas encontrados como producto del análisis de contenido \\
\hline Categoría & No. de artículos \\
\hline Competencias transversales para la empleabilidad & 12 \\
\hline Equidad y empleabilidad & 4 \\
\hline Fomento de la empleabilidad & 10 \\
\hline Internacionalización (movilidad estudiantil) y empleabilidad & 3 \\
\hline Programas educativos y empleabilidad & 10 \\
\hline Relación universidad-mercado laboral & 7 \\
\hline Total de artículos & $\mathbf{4 6}$ \\
\hline
\end{tabular}

Fuente: Elaboración propia.

A continuación, se explican los hallazgos del estudio por cada categoría de tema encontrado como parte del análisis de contenido. Se incluyen ejemplos de algunos de los artículos incluidos en la muestra para puntualizar los hallazgos. Las referencias de los artículos de la muestra que se citan a continuación se encuentran en el anexo marcados con asterisco $(*)$.

\section{C'ompetencias transverssiles parlia lia empleabilitidad}

Doce de los 46 artículos en la muestra abordaron el tema de competencias transversales para la empleabilidad. Los artículos en esta categoría sugieren que existen investigadores interesados en la evaluación y análisis de competencias para la empleabilidad de los jóvenes profesionistas. Por ejemplo, Hernández Fernaud et al. (2011) construyeron un cuestionario para medir las escalas de empleabilidad percibida y autoeficacia en estudiantes de profesional. Los autores concluyeron que la percepción de los estudiantes respecto a sus niveles de empleabilidad y su percepción de eficacia respecto a la búsqueda de empleo están relacionadas de manera significativa. La aportación principal de estos autores radica en la identificación de una escala que se puede utilizar como guía para educadores y estudiantes en busca de competencias específicas a desarrollar. Con la información que deriva de este tipo de estudios, personal docente y administrativo en IES logran conocer mejor el perfil de sus estudiantes en función de su propia percepción para desempeñarse en el mercado laboral e implementar estrategias educativas para realizar los ajustes que correspondan.

Como ejemplo de hallazgos aplicables para el desarrollo de una estrategia educativa que promueva la empleabilidad, Gualteros et al. (2011) confirmaron la importancia de introducir el desarrollo de habilidades informativas en instituciones educativas como estrategia para preparar a los estudiantes para las demandas del mercado laboral. Tanto el trabajo de Hernández Fernaud et al. (2011) como el de Gualteros et al. (2011), y los demás artículos en esta categoría, aportan literatura sobre empleabilidad entre personas con estudios universitarios desde la perspectiva de competencias. Dichas competencias tienden a ser genéricas o transversales, como las informativas mencionadas anteriormente, que son aplicables para cualquier giro 0 industria. 


\section{Elpuitad y empleatililidid}

Cuatro de los 46 artículos en la muestra abordaron el estudio de la empleabilidad desde la perspectiva de la equidad e inclusión. Este tipo de estudios normalmente buscan contribuir a cerrar la brecha de género, nivel socio-económico, etnicidad, orientación sexual o creencia religiosa en la educación y en el trabajo. En algunos países, el tema de inclusión de género comienza a cobrar mayor importancia. En un estudio realizado en México, Contreras-Cueva y Rubio González (2014) concluyeron que la participación de la mujer profesionista en el mercado laboral se incrementa, incluso en áreas de ingeniería donde tradicionalmente han dominado los hombres. Entre los trabajos encontrados en el análisis de contenido del presente estudio se encuentra la aportación de Formichella y London (2013), quienes argumentaron que existen diferencias en términos de oportunidades de empleo para ciertos alumnos y que algunas de estas inequidades se perpetúan a través de la educación.

Esta información puede ser útil para docentes respecto a la forma en que abordan el tema de la empleabilidad con sus estudiantes que tienen antecedentes diversos. En este sentido, Macedo Ziliotto y Laureano dos Santos (2015) entrevistaron a mujeres en el mercado laboral para conocer sus experiencias respecto a discriminación por género. Las mujeres participantes en el estudio reportaron que su experiencia en la universidad les ayudó a prepararse para el mercado laboral, pero que son susceptibles a prácticas de discriminación por razones de género en el trabajo. Este estudio sugiere que existe necesidad de continuar los esfuerzos por integrar la investigación, docencia y la industria. Esto se puede facilitar mediante vinculación entre IES y empresas locales donde se coloquen sus egresados.

\section{Fomento de la empleabilididad}

Diez de los 46 artículos en la muestra documentaron acciones llevadas a cabo por académicos y administradores educativos para fomentar el empleo y la empleabilidad entre sus estudiantes.

Por ejemplo, Romero Fernández y Álvarez Gavilanes (2015) investigaron las perspectivas de egresados de la carrera de Administración de Empresas de la Universidad Regional Autónoma de los Andes y de profesores y empleadores de la región para identificar el nivel de congruencia entre las competencias que desarrollan los estudiantes y la demanda del mercado laboral. Los autores concluyeron que las competencias que se promueven en el centro universitario y las que demanda el mercado laboral están desarticuladas, lo cual resulta en un alto índice de desempleo entre los egresados.

Con esta información, los docentes, administradores y empleadores pueden trabajar en equipo para desarrollar programas educativos que fomenten las competencias que demanda el mercado laboral. Con este tipo de estrategias, se aborda el problema de desempleo entre estudiantes recién egresados y la falta de capital humano competente en las empresas. En concreto, la aplicación de este tipo de estudios radica en el desarrollo de nuevas cartas descriptivas, planes de estudio, programas académicos

230 y proyectos cocurriculares. 


\section{Internaccionalizización y empleathilididal}

Tres de los 46 estudios en la muestra abordaron el tema de la empleabilidad desde la perspectiva de la internacionalización y movilidad estudiantil. Las interrogantes planteadas en este tipo de estudio parten de dudas sostenidas por docentes respecto a la relevancia de experiencias internacionales entre estudiantes respecto a su capacidad para colocarse en un empleo. A través de estos artículos, dichas interrogantes son abordadas. Por ejemplo, Geldres, Ribeiro de Almeida y Flander (2015) examinaron la percepción de empleadores en Brasil respecto a la movilidad internacional de estudiantes. Los autores concluyeron que los empleadores valoran las competencias que desarrollan los estudiantes durante sus experiencias de trabajo y estudio en el extranjero. Asimismo, Barraycoa Martínez y Lasaga Millet (2012) examinaron las experiencias de estudiantes que realizaron una estancia profesional durante sus estudios en la universidad. Los autores concluyeron que los alumnos no necesariamente consideran la movilidad internacional como una forma de desarrollar competencias para la empleabilidad. Sin embargo, al iniciar el proceso de inserción laboral, después de concluir su experiencia internacional, los alumnos aprecian el efecto favorable de dicha práctica en la inserción laboral, especialmente en términos del dominio de un segundo idioma. Por tanto, las experiencias de movilidad internacional fomentan competencias que permiten a los estudiantes colocarse con mayor facilidad en un empleo.

\section{Progritmitis educrativos y empleabilitidad}

Diez de los 46 artículos en la muestra fueron diseñados para examinar la efectividad de programas educativos curriculares o cocurriculares que se implementan para ayudar a sus estudiantes a preparase para la empleabilidad. Por ejemplo, Foncubierta Rodríguez, Perea Vicente y González Siles (2016) trabajaron con un grupo de expertos para desarrollar un perfil de egreso de estudiantes universitarios enfocado al desarrollo de competencias para la empleabilidad. Los autores reportaron que dicho perfil se constituye de competencias genéricas, técnicas y de gestión empresarial. Asimismo, Prieto Serrano, Manzano-Soto y Villalón Martínez (2017) analizaron las experiencias y resultados de autoevaluaciones de individuos involucrados en programas de prácticas profesionales virtuales en función de su preparación para el empleo. Los autores concluyeron que la modalidad de prácticas profesionales virtuales contribuye al desarrollo de competencias para la empleabilidad. Por otra parte, Tarrats Pons et al. (2018) examinaron las experiencias relacionadas con la implementación de un programa de educación en alternancia en cuatro universidades participantes en España y Francia. Los autores concluyeron que la alternancia, como estrategia de enseñanza-aprendizaje, es efectiva para la promoción de la empleabilidad de estudiantes en un contexto transfronterizo.

En estos ejemplos se alcanzan a apreciar algunas iniciativas de vinculación, integración de tecnologías de la comunicación y adopción de enfoques educativos no tradicionales para fomentar la empleabilidad de los estudiantes. Esto sugiere que quienes se dedican a la educación están dispuestos a intentar diferentes estrategias para 
fomentar la empleabilidad. La tolerancia a la ambigüedad y la disposición favorable hacia la prueba y error dan espacio para que las IES intenten implementar nuevas formas de preparar a sus estudiantes. En este sentido, el trabajo de investigación de quienes dirigen este tipo de esfuerzo es fundamental para documentar las iniciativas de fomento a la empleabilidad, sus procesos y resultados.

\section{Relación entre la universidad y el merctido laborial}

Siete de los 46 estudios en la muestra analizaron la articulación entre la demanda del mercado laboral y la educación que reciben los estudiantes en las universidades. Por ejemplo, del Cerro Velázquez y Ramón Cano (2017) analizaron información de egresados universitarios en España y concluyeron que existe un desajuste entre la formación universitaria y las competencias que demanda el mercado laboral. Asimismo, Ramírez del Río y Garrido Casas (2011) analizaron la relación entre la cantidad de horas destinadas al desarrollo de competencias para la empleabilidad durante la formación académica profesional de individuos en España y su desempeño en el mercado laboral. Los autores encontraron evidencia de que existe una relación positiva, especialmente en términos de emprendimiento en pequeñas y medianas empresas. Con hallazgos como estos, los administradores y docentes en IES pueden justificar iniciativas para definir la relación entre sus instituciones educativas y los mercados que sirven, todo en función de los perfiles de ingreso y egreso de sus estudiantes.

En respuesta a las tres preguntas de investigación planteadas en este estudio, se hace notar que las competencias transversales o genéricas son las que mejor se alinean con los hallazgos de los estudios revisados. Competencias relacionadas con el desarrollo de habilidades informativas, idiomas y gestión aparecen en la literatura como enfoque de las estrategias de aprendizaje en IES y que son valoradas por empleadores. Asimismo, experiencias de movilidad internacional, prácticas profesionales, educación en alternancia y tomar cursos en línea promueven la empleabilidad de los estudiantes y egresados. Finalmente, el análisis de contenido confirma que los docentes que documentan sus estrategias educativas en congruencia con el enfoque de SoTL encuentran espacios en revistas arbitradas. Esto sugiere que las actividades de docencia, servicio e investigación se pueden articular como parte de un mismo objetivo. Asimismo, es razonable asumir que los editores de revistas arbitradas se encuentran abiertos a publicar artículos que se desarrollan con base en el enfoque propuesto por Boyer et al. (2016). Esta afirmación se puede evaluar mediante investigaciones futuras.

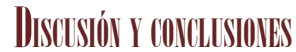

Las ideas de Boyer et al. (2016) sugieren que las IES den mayor prioridad al desarrollo de profesionales capaces de contribuir a la sociedad de forma positiva. Sin embargo, su planteamiento no implica que los docentes abandonen su interés por la 232 investigación para enfocarse más en la docencia o la vinculación. Su argumento se 
centra en la articulación entre el trabajo de docencia, servicio e investigación para beneficiar lo más posible a los estudiantes. Esta postura ha sobrevivido con el tiempo porque su lógica es congruente con los valores de las personas que se dedican a la educación (Boyer et al., 2016). En este estudio, se sostiene el supuesto de que los docentes y el personal administrativo en las IES están dispuestos a llevar a cabo iniciativas para mejorar la relevancia de sus programas y facilitar la transición de los estudiantes que terminan sus estudios universitarios y se integran al mercado laboral como profesionistas.

Los 46 artículos examinados en este estudio mostraron seis categorías de temas que representan estrategias educativas utilizadas para el fomento de la empleabilidad (tabla 1). En estos artículos se alcanza a apreciar cómo se articulan prácticas docentes y programas educativos con la investigación. Gracias a esto se facilita el trabajo de difusión de mejores prácticas educativas que ayudan a acotar la brecha entre las IES y el mercado laboral. Las implicaciones de esto incluyen mejores oportunidades para facilitar la toma de decisiones en términos de mecanismos para diseño curricular, medición del aprendizaje y rendición de cuentas. Potencialmente, la articulación entre la docencia, el servicio y la investigación pueden facilitar la creación de programas de vinculación, prácticas profesionales, bolsa de trabajo, movilidad estudiantil y procesos de acreditación. Todos estos elementos de la educación tienen implicación para la empleabilidad. Corresponde a los líderes educativos evaluar sus prácticas educativas actuales, revisar los índices de colocación de sus egresados y realizar los ajustes pertinentes para mejorar su desempeño en el rubro de la empleabilidad.

Es recomendable desarrollar futuras investigaciones en función de la propuesta de Boyer para estudiar el tema de la empleabilidad en diferentes lugares del mundo. Como se mencionó en este documento, la búsqueda de artículos se llevó a cabo mediante el uso de palabras en español, dando un sesgo hacia publicaciones en países de habla hispana, tomando en cuenta que este tipo de investigación está muy desarrollado en publicaciones en inglés. A pesar de que fue posible trabajar con una muestra de 46 artículos, la mayor parte de estos se encontraron en revistas españolas. Tomando en cuenta esta situación, se recomienda incentivar prácticas docentes y proyectos de investigación educativa enfocados en la empleabilidad en países de Latinoamérica. Esto se facilita mediante la organización de congresos bajo la línea de investigación de empleabilidad y temas relacionados, convocatorias de números especiales en revistas arbitradas sobre empleabilidad y talleres y coloquios institucionales sobre el tema. Asimismo, como forma de superar la limitante en el diseño de este estudio, aquella que resulta de utilizar únicamente la base de datos EBSCO Host como fuente de artículos, se recomienda la integración de publicaciones indexadas en otras bases de datos académicas.

A manera de conclusión, las seis categorías de temas sobre empleabilidad analizadas en este trabajo representan líneas de investigación viables para docentes y administradores educativos interesados en promover la empleabilidad entre sus estudiantes y avanzar la propuesta de Boyer (SoTL). La existencia de estos trabajos sirve como evidencia de que es posible encontrar revistas académicas y editores interesados en promover este tipo de investigaciones. Por tanto, docentes y docentes-investigadores tienen un claro incentivo para implementar estrategias educativas enfocadas 
en la empleabilidad de sus estudiantes, documentar sus experiencias y compartir sus hallazgos mediante publicaciones académicas. Para esto, es recomendable que revistas arbitradas comuniquen su intención de publicar artículos desarrollados con base en las premisas de SoTL para incentivar el desarrollo investigaciones que parten de actividades de docencia o servicio. Este proceso se facilita considerablemente cuando los administradores académicos apoyan a los docentes-investigadores para que implementen estrategias educativas conducentes a promover la empleabilidad mediante docencia, servicio e investigación.

\section{Repreperillis}

Arias López, K., Ortiz Cáceres, I. y Fernández Lobos, G. (2018). Articulación de itinerarios formativos en la educación superior técnico profesional. Perfiles Educativos, 11(160), 174190.

Backhoff Escudero, E., Guevara Niebla, G., Hernández Uralde, J. y Sánchez Moguel, A.E. (2018). El aprendizaje al término de la educación media superior en México. El Cotidiano. Revista de la Realidad Mexicana Actual, 33(208), 7-19.

Banathy, B.H. (1999). Systems thinking in higher education: Learning comes to focus. Systems Research \& Behavioral Science, 16(2), 133-145.

Bernstein, J.L. (2018). Finding moments of meaning in undergraduate education: How the scholarship of teaching and learning can help. International Journal for the Scholarship of Teaching \& Learning, 12(1), 1-8.

Bonney, K.M. (2018). Students as partners in the scholarship of teaching and learning. International Journal for the Scholarship of Teaching \& Learning, 12(2), 1-5.

Bowen, G. (2010). Service learning in the scholarship of teaching and learning: Effective practices. International Journal for the Scholarship of Teaching \& Learning, 4(2), 1-15.

Boyer, E.L., Moser, D., Ream, T.C. y Braxton, J.M. (2016). Scholarship reconsidered: Priorities of the professoriate (expanded edition). Jossey-Bass.

Caballero, K. y Bolívar, A. (2015). El profesorado universitario como docente: hacia una identidad profesional que integre docencia e investigación. Revista de Docencia Universitaria, 13(1), $57-77$.

Chadha, D. y Toner, J. (2017). Focusing in on employability: Using content analysis to explore the employability discourse in UK and USA Universities. International Journal of Educational Technology in Higher Education, 14(1), 1-26. https://dx.doi.org/10.1186/s41239-017-0071-0

Contreras-Cueva, A.B. y Rubio González, J.A. (2014). Las tendencias del capital humano en México: un análisis de la trayectoria de la educación por género 2005-2012. Entreciencias: Diálogos en la Sociedad del Conocimiento, 2(3), 67-80.

Cunningham, J. (2016). Genres of underemployment: A dialogical analysis of college graduate underemployment. Qualitative Research in Education, 5(1), 1-24.

Davis, A., Dent, E. y Wharff, D. (2015). A conceptual model of systems thinking leadership in community colleges. Systemic Practice \& Action Research, 28(4), 333-353.

de la Luz Tovar, C. y Díaz González, E. (2010). Dispersión del ingreso y demanda de educación media superior y superior en México. Análisis Económico, 25(58), 99-122.

Dibenedetto, C.A. y Myers, B.E. (2016). A conceptual model for the study of student readiness in the 21st century. NACTA Journal, 60, 28-35.

Divan, A., Ludwig, L.O., Matthews, K.E., Motley, P.M. y Tomljenovic-Berube, A.M. (2017). Survey of research approaches utilized in the scholarship of teaching and learning publications. Teaching \& Learning Inquiry, 5(2), 16-29.

234 Dunnion, J. y O'Donovan, B. (2014). Systems thinking and higher
method. Systemic Practice \& Action Research, 27(1), 23-37. 
Eliška, K. (2016). Risk factors of young graduates in the competitive E.U. labor market at the end of the current economic crisis. Journal of Competitiveness, 7(3), 38-51.

Fernández Fassnacht, E. (2017). Una mirada a los desafíos de la educación superior en México. Journal Educational Innovation / Revista Innovación Educativa, 12(74), 183-207.

Gervilla Castillo, E. (2004). Buscando valores: el análisis de contenido axiológico. Perfiles Educativos, 26(103), 95-110.

Haasler, S.R. (2013). Employability skills and the notion of self. International Journal of Training \& Development, 17(3), 233-243.

Hualde, A. y Serrano, A. (2005). La calidad del empleo de asalariados con educación superior en Tijuana y Monterrey. Revista Mexicana de Investigación Educativa, 10(25), 345-374.

Huisman, J. y Currie, J. (2004). Accountability in higher education: Bridge over troubled water? Higher Education, 48(4), 529-551.

Humphreys, D. (2014). Employment outcomes in the four-year sector: The value of liberal arts degrees. Change, 46(3), 64-66.

Instituto Nacional de Estadística y Geografía, INEGI. (2018). Encuesta Nacional de Ocupación y Empleo. Recuperado de http://www.inegi.org.mx/lib/olap/consulta/general_ver4/ MDXQueryDatos_colores.asp?c=

Ison, R. (1999). Applying systems thinking to higher education. Systems Research \& Behavioral Science, 16(2), 107-112.

Jiménez Moreno, J.A. (2017). La evaluación de los egresados de formación profesional en México: reflejo de la implementación de la política de competitividad en la educación superior. Education Policy Analysis Archives, 25(43-52), 1-18.

Kezar, A. (2005). What campuses need to know about organizational learning and the learning organization. New Directions for Higher Education 2005, (131), 7-22.

Kreber, C. (2002). Teaching excellence, teaching expertise, and the scholarship of teaching. Innovative Higher Education, 27(1), 5-23.

Licona Michel, Á. y Rangel Delgado, J.E. (2013). Pilares de la competitividad, educación superior, nuevas tecnologías y empleo en Corea del Sur y México. Análisis Económico, 28(69), 79-108.

López Aguado, M. (2018). La evaluación de la calidad de títulos universitarios. Dificultades percibidas por los responsables de los sistemas de garantía de calidad. Educación XX1, 21(1), 263-283.

Martínez Lobatos, L. (2014). Currículo y vinculación: una relación socioeducativa aplazada para la formación profesional. Sinéctica, (43), 1-21.

Mathany, C., Clow, K.M. y Aspenlieder, E.D. (2017). Exploring the role of the scholarship of teaching and learning in the context of the professional identities of faculty, graduate students, and staff in higher education. Canadian Journal for the Scholarship of Teaching \& Learning, $8(3), 1-17$.

Milner, S., Cousins, W. y McGowan, I. (2016). Does all work and no play make a dull graduate? Perceptions of extra-curricular activities and employability. Journal of Perspectives in Applied Academic Practice, 4(1), 13-18.

Moctezuma, P., Ocegueda, M.T., Mungaray, A., Ocegueda, J.M. y Estrella, G. (2013). Cobertura con calidad a través de la reorganización institucional en la educación superior de Baja California. Frontera Norte, 25(49), 87-108.

Pfleger, R.H., Wilson, T.S., Welner, K. y Bibilos, C. (2018). Measuring opportunity: Redirecting education policy through research. Education Policy Analysis Archives, 26(72/73), 1-27.

Ponnuswamy, I. y Lysander, H. (2016). Impact of learning organization culture on performance in higher education institutions. Studies in Higher Education, 41(1), 21-36.

Saldaña, J. (2016). The coding manual for qualitative researchers ( $3^{\mathrm{a}}$ ed.). Thousand Oaks, California: Sage.

Senge, P., Cambron-McCabe, N., Lucas, T. (2012). Schools that learn: Updated and revised. Nueva York: Crown Business. 
Sueyoshi, A. y Rangel Delgado, J.E. (2016). La convergencia de las políticas de educación superior y las políticas de empleo en los mercados laborales de Japón y México. Portes: Revista Mexicana de Estudios sobre la Cuenca del Pacifico, 10(20), 81-103.

Udall, J., Forrest, D. y Stewart, K. (2015). Locating and building knowledges outside of the academy: Approaches to engaged teaching at the University of Sheffield. Teaching in Higher Education, 20(2), 158-170. https://dx.doi.org/10.1080/13562517.2014.966237

van der Meulen, B. (2002). Europeanization of research and the role of universities: An organizational-cultural perspective. Innovation: The European Journal of Social Sciences, 15(4), 341-355.

White, M.D. y Marsh, E.E. (2006). Content analysis: A flexible methodology. Library Trends, $60(1), 22-45$.

Wolff, R. y Booth, M. (2017). Bridging the gap: Creating a new approach for assuring 21st century employability skills. Change: The Magazine of Higher Learning, 49(6), 51-54. https://dx.doi. org/10.1080/00091383.2017.1399040

Yingqiang, Z. y Yongjian, S. (2016). Quality assurance in higher education: Reflection, criticism, and change. Chinese Education \& Society, 49(1/2),7-19.

\section{AIEXO

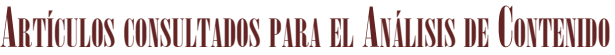

Aliaga, C. y Schalk, A. (2010). E2: Empleabilidad temprana y emprendimiento. Dos grandes desafios en la formación superior en Chile. Calidad en la Educación, (33), 319-337.

Almanza Jiménez, R. y Vargas-Hernández, J.G. (2015). Las competencias profesionales y su relación con la empleabilidad de los ingenieros en gestión empresarial egresados del ITLAC. Revista Electrónica Gestión de las Personas y Tecnologías, 8(22), 17-28.

Barraycoa, M.J. y Lasaga Millet, O. (2012). Competencias desarrolladas en una experiencia internacional y empleabilidad: una perspectiva de género. @Tic.Revista D'innovació Educativa, (8), 1-11.*

Brown, R. (2015). La evaluación auténtica: el uso de la evaluación para ayudar a los estudiantes a aprender. RELIEVE-Revista Electrónica de Investigación y Evaluación Educativa, 11(2), $1-10$.

Caballero Fernández, G., López-Miguens, M.J. y Lampón, J.F. (2014). Spanish universities and their involvement with the employability of graduates. Revista Española de Investigaciones Sociológicas, (146), 23-45.

Cuesta Cambra, U. y Mañas Viniegra, L. (2016). Integración de la realidad virtual inmersiva en los grados de comunicación. Icono, 14(2), 1-21.

Dapía Conde, M.D. y Reyes Fernández González, M. (2016). La búsqueda activa de empleo en el colectivo universitario: conocimientos y prácticas. Revista Española de Pedagogía, 74(265), $517-542$

De la Torre Jr., D. y Wells, R. (2014). Evolving statewide transfer policies: Persistent efforts in tension with workforce development among Massachusetts community colleges. Education Policy Analysis Archives, 12(20), 1-28.

del Arco Bravo, I. y Enciso Rodríguez, P. (2011). Valoración de las competencias instrumentales de los titulados universitarios: Estudio comparativo. Bordón, 63(3), 91-105.

del Cerro Velázquez, F. y Ramón Cano, F.J. (2017). Compromiso y empleabilidad de los recién titulados de formación profesional. Conclusiones para un rediseño de la modalidad formativa. RED-Revista de Educación a Distancia, (54), 1-15.*

Eizagirre Sagardia, A., Altuna Urdin, J. y Fernández Fernández, I. (2017). Prácticas de éxito en el desarrollo de competencias transversales en centros de formación profesional del País Vasco. Revista Española de Pedagogía, 75(267), 293-308. 
Fernández Solo de Zaldívar, I. (2017). Mejora de competencias: introducción de la gestión de calidad en nuevas metodologías educativas. Profesorado: Revista de Curriculum y Formación del Profesorado, 21(2), 279-308.

Fitó Bertran, À., Martínez Argüelles, M.J. y Moya Gutiérrez, S. (2014). El perfil competencial de los graduados de administración y dirección de empresas en línea: una visión desde el mercado de trabajo. RUSC: Revista de Universidad y Sociedad del Conocimiento, 11(2), 1326.

Flechas Chaparro, N. y Juárez Acosta, F. (2017). La prosocialidad en ambientes virtuales de aprendizaje y la empleabilidad. Revista Virtual Universidad Católica del Norte, (51), 124142.

Foncubierta Rodríguez, M.J., Perea Vicente, J.L. y González Siles, G. (2016). Una experiencia en la vinculación universidad-empresa: el proyecto COGEMPLEO de la Fundación Campus Tecnológico de Algeciras. Educación XX1, 19(1), 201-225.*

Formichella, M.M. y London, S. (2013). Empleabilidad, educación y equidad social. Revista de Estudios Sociales, (47), 79-91.*

García Blanco, M. y Cárdenas Sempértegui, E.B. (2018). La inserción laboral en la educación superior. La perspectiva latinoamericana. Educación XX1, 21(2), 323-347.

Geldres, V.V., Ribeiro de Almeida, M.I. y Flander, A. (2015). Movilidad internacional de estudiantes y empleabilidad. La visión de los empleadores. Revista Iberoamericana de Ciencia, Tecnología y Sociedad, 10(30), 113-141.*

Gijón Puerta, J. y Crisol Moya, E. (2012). La internacionalización de la educación superior. El caso del Espacio Europeo de Educación Superior. Revista de Docencia Universitaria, 10(1), 389-414.

Gil Vega, L. (2017). El proceso de Bolonia y la escuela nueva: un análisis comparado de convergencias y divergencias. Educação, Sociedade \& Culturas, (51), 119-136.

González Pérez, A. (2015). Claves pedagógicas para la mejora de la calidad del EEES. Educatio Siglo XXI, 33(1), 259-276.

Gualteros, N., Marciales Vivas, G.P., Cabra Torres, F. y Mancipe Flechas, E. (2011). Inserción sociolaboral juvenil y competencias informacionales en la educación superior: desarrollos, tensiones y desafíos. Signo y Pensamiento, 31(59), 102-119.*

Gutiérrez Ossa, J.A. (2013). Universidad, empresa y Estado frente a la empleabilidad de los trabajadores de la ciencia en Colombia. Ecos de Economía, 17(36), 69-98.

Hernández Fernaud, E., Ramos-Sapena, Y., Negrín, F. (2011). Empleabilidad percibida y autoeficacia para la búsqueda de empleo en universitarios. Revista de Psicología del Trabajo $y$ de las Organizaciones, 37(2), 131-142.*

Macedo Ziliotto, D. y Laureano dos Santos, L. (2015). Histórias de trabalho de mulheres negras com ensino superior. Revista Latinoamericana de Geografia e Gênero, 6(2), 71-91.*

Martín Villarroya, R. y Ramos-Villagrasa, P.J. (2017). Competencias clave para la empleabilidad de los egresados universitarios: un análisis en la Universidad de Zaragoza. Acciones e Investigaciones Sociales, (37), 215-237.

Monllau Jaques, T.M. y Rodríguez Ávila, N. (2015). Importancia de la utilización de las TIC como garantes de la agilidad, eficiencia y comunicación entre empresa, universidad y estudiante: experiencia de la Facultad de Ciencias Económicas y Empresariales de la Universidad Pompeu Fabra. Intangible Capital, 11(4), 577-588.

Moreno Romani, P.R. (2012). Calidad una decisión estratégica en la educación superior. Revista Politécnica, 8(14), 9-15.

Moreno Mínguez, A. (2015). La empleabilidad de los jóvenes en España: explicando el elevado desempleo juvenil durante la recesión económica. Revista Internacional de Investigación en Ciencias Sociales, 11(1), 3-20.

Oliver, A., Galiana, L. y Gutiérrez Benet, M. (2016). Diagnóstico y políticas de promoción del emprendimiento en estudiantes. Anales de Psicología, 32(1), 183-189. 
Ordóñez, J.L., Figuera Gazo, P. y Torrado Fonseca, M. (2017). Desarrollo de la empleabilidad y gestión personal de la carrera de graduados en pedagogía. Revista Española de Orientación y Psicopedagogía, 28(2), 46-60.

Passarini, J., Rodríguez, B. y Borlido, C. (2016). Impacto de un cambio curricular en la empleabilidad de los graduados veterinarios de la Universidad de la República de Uruguay. Revista Cubana de Educación Superior, 35(1), 64-74.

Paterson, R. (2017). Because sometimes your failures can also teach you certain skills: Lecturer and student perceptions of employability skills at a transnational university. Qualitative Research in Education, 6(3), 241-275.

Paz Rodríguez, F., Betanzos-Díaz, N. y Uribe-Barrera, N. (2014). Expectativas laborales y empleabilidad en enfermería y psicología. Aquichan, 14(1), 67-78.

Prieto Serrano, D., Manzano-Soto, N. y Villalón Martínez, M.J. (2017). Prácticas profesionales virtuales como estrategia de empleabilidad: el caso de la UNED. Revista Española de Orientación y Psicopedagogía, 28(2), 122-138.*

Salinas, P. y Romaní, G. (2017). Proyección laboral de las estudiantes mujeres en carreras mineras en la educación superior chilena. Formación Universitaria, 10(3), 31-48.

Ramírez del Río, A. y Garrido Casas, J. (2011). Evaluación del impacto del esfuerzo formativo en la empleabilidad de los trabajadores en el contexto del modelo formativo tripartito español. RELIEVE-Revista Electrónica de Investigación y Evaluación Educativa, 17(2), 1-29.*

Rodríguez Garcés, C. y Castillo Riquelme, V. (2014). Empleabilidad, ingresos y brechas: un análisis comparativo de los procesos de inserción laboral en Chile. Orientación y Sociedad, (14), 1-19.

Rojas Rojas, D. (2014). Impacto en la situación laboral de egresados de la educación presencial y virtual: estudio comparativo. Latin American Journal of Content \& Language Integrated Learning, 17(2), 339-354.

Romero Fernández, A. y Álvarez Gavilanes, J. (2015). Diagnóstico de la inserción laboral de graduados de administración de empresas de la Universidad Regional Autónoma de los Andes. Pedagogía Universitaria, 20(2), 58-76.*

Rosado May, F.J. (2017). Formación universitaria intercultural para indígenas mayas de Yucatán, México. Antropológica, 35(39), 215-242.

Suárez Lantarón, B. (2013). Servicios de orientación profesional universitarios: estudio descriptivo. Revista de Docencia Universitaria, 11(2), 27-42.

Suárez Lantarón, B. (2016). Empleabilidad: análisis del concepto. Revista de Investigación en Educación, 14(1), 67-84.

Tarrats Pons, E., Arimany Serrat, N. y Arando Lasagabaster, S. (2018). Educación superior en alternancia transfronteriza entre Francia y España. Educación XX1, 21(2), 275-300.*

Verano Tacoronte, D., Bolívar Cruz, A. y González-Betancor, S.M. (2015). Self-assessment: A critical competence for industrial engineering. Dyna, 82(194), 130-138. 